\title{
Modulation of Rabbit Platelet Aggregation and Calcium Mobilization by Platelet Cholesterol Content
}

\author{
Mitsuya SHIRAISHI ${ }^{1)}$, Eisuke TANI ${ }^{1)}$ and Atsushi MIYAMOTO ${ }^{1) *}$ \\ ${ }^{1)}$ Department of Veterinary Pharmacology, Faculty of Agriculture, Kagoshima University, 1-21-24 Korimoto, Kagoshima 890-0065, \\ Japan
}

(Received 31 August 2009/Accepted 24 October 2009/Published online in J-STAGE 1 December 2009)

\begin{abstract}
Hypercholesterolemia is one of the major contributing factors in atherosclerosis and the development of cardiovascular disease. Platelets from hypercholesterolemic rabbit have an increased cholesterol content and a hypersensitivity to endogenous aggregating agonists. Although rabbit has been widely used in studies of hypercholesterolemia, the precise role of platelet cholesterol in rabbit platelet activation has not been studied. In the present study, to determine the direct role of cholesterol on rabbit platelet activation, we examined the effect of in vitro modulation of cholesterol content on platelet activation. Cholesterol-depleted rabbit platelets by the treatment with methyl- $\beta$-cyclodextrin showed decreased platelet aggregation by physiological agonists such as thrombin, adenosine diphosphate, and collagen. The inhibition of thrombin-induced aggregation in cholesterol-depleted platelets was restored by cholesterol repletion in platelets. The cholesterol depletion also inhibited $\mathrm{Ca}^{2+}$ mobilization, which plays a pivotal role in the platelet activation induced by physiological agonists. We showed that the $\mathrm{Ca}^{2+}$ influx pathway is strongly suppressed by cholesterol depletion more than $\mathrm{Ca}^{2+}$ release from intracellular $\mathrm{Ca}^{2+}$ stores in platelets stimulated with thrombin. Furthermore, platelet aggregation induced by PMA, a potent protein kinase $\mathrm{C}$ activator, was also depressed by cholesterol depletion. On the other hand, cholesterol enrichment in platelets augmented thrombininduced aggregation and $\mathrm{Ca}^{2+}$ mobilization. These findings suggest that cholesterol plays a critical role in regulating rabbit platelet activation, and provides fundamental information regarding hypercholesterolemia-mediated effects on cells in the rabbit model.

KEY WORDS: calcium, cholesterol, platelet.
\end{abstract}

Platelets play an essential role in hemostasis. At sites of vascular injury, circulating platelets adhere to exposed subendothelial collagen with the aid of von Willebrand factor and become activated. Activated platelets undergo shape change and spreading; they also release factors such as adenosine diphosphate (ADP) and thromboxane $\mathrm{A}_{2}\left(\mathrm{TXA}_{2}\right)$ that activate additional platelets. In contrast, thrombin is generated during blood coagulation and acts as a potent platelet activator. Activated platelets result in platelet aggregation to form a haemostatic plug at the injury site. It has become increasingly evident that platelets not only play an important role in hemostasis but also are involved in pathological conditions such as thrombosis and atherosclerosis [15, 33].

Hypercholesterolemia is one of the major contributing factors in atherosclerosis and the development of cardiovascular disease [14, 29]. Platelets from hypercholesterolemic humans have increased cholesterol content and hypersensitivity to endogenous aggregating agonists [9, 21, 25]. In familial hypercholesterolemic patients, cholesterol-lowering treatment with statin, an HMG-CoA reductase inhibitor, decreases the platelet cholesterol content and platelet sensitivity to the aggregating agonists such as ADP and collagen $[9,17,18]$. On the other hand, in vitro cholesterol depletion from the human platelet membrane decreases platelet sensitivity to the agonists such as ADP, collagen, and thrombin, and reduces the release of $\mathrm{TXA}_{2}$ and $\mathrm{ADP}[1,7,20,30]$. In

\footnotetext{
* Correspondence to: Miyamoto, A., Department of Veterinary Pharmacology, Faculty of Agriculture, Kagoshima University, 1-21-24 Korimoto, Kagoshima 890-0065, Japan. e-mail: miyamoto@agri.kagoshima-u.ac.jp
}

contrast, in vitro cholesterol enrichment of human platelets results in hypersensitivity to agonists $[13,26]$. These previous studies have indicated that the cholesterol content directly influences human platelet activation, and suggest an important role for hypersensitive platelets in the development of thrombosis and atherosclerosis.

The rabbit has been widely used in studies of atherosclerosis because, in addition to its size and easy manipulation, mild cholesterol supplementation in the rabbit leads to hypercholesterolemia and subsequent atherosclerosis [12, 23, 35]. Furthermore, a spontaneously hypercholesterolemic model, Watanabe heritable hyperlipidemic rabbits (WHHL) [34], is available and has been utilized to understand human familiar hypercholesterolemia and atherosclerosis. Although an increased cholesterol content and hypersensitivity of platelets from hypercholesterolemic rabbit has been reported $[3,28]$, the precise role of platelet cholesterol in rabbit platelet activation has not been studied. In the present study, to determine the direct role of cholesterol in rabbit platelet activation, we examined the effects of in vitro modulation of cholesterol content on platelet aggregation and $\mathrm{Ca}^{2+}$ mobilization induced by physiological agonists such as thrombin, collagen, or ADP.

\section{MATERIALS AND METHODS}

Materials: Methyl- $\beta$-cyclodextrin (M $\beta \mathrm{CD})$, cholesterol, ADP, thrombin, phorbol 12-myristate 13-acetate (PMA), apyrase, and bovine serum albumin (BSA) were obtained from Sigma (St. Louis, MO, U.S.A). Collagen was from 
Nycomed Pharma (Oslo, Norway). Prostaglandin $E_{1}$ $\left(\mathrm{PGE}_{1}\right)$ was purchased from Cayman Chemical (Ann Arbor, MI, U.S.A.). Fura-PE3/AM was purchased from Teflabs (Austin, TX, U.S.A.). Aspirin and the cholesterol kit (cholesterol E-test wako) were from Wako Pure Chem (Osaka, Japan). Protein assay CBB solution was from Nacalai tesque (Kyoto, Japan).

Platelet preparation: Blood was obtained from the marginal ear vein of male Japanese white rabbit $(2.5-3.5 \mathrm{~kg}$ ), and was mixed with a one-tenth volume of $3.8 \%$ sodium citrate as anticoagulant. Platelet-rich plasma (PRP) was prepared by centrifugation of blood at $160 \mathrm{~g}$ for $10 \mathrm{~min}$ at room temperature. The PRP was centrifuged at $670 \mathrm{~g}$ for $10 \mathrm{~min}$ in the presence of $1 \mu \mathrm{M} \mathrm{PGE}$ and 0.05 unit $/ \mathrm{m} l$ apyrase to prevent platelet activation during the centrifugation. The pelleted platelets were resuspended with Hepes-Tyrode's buffer (20 mM Hepes, $0.34 \mathrm{mM} \mathrm{NaH}{ }_{2} \mathrm{PO}_{4}, 137 \mathrm{mM} \mathrm{NaCl}, 1$ $\mathrm{mM} \mathrm{MgCl}, 2.7 \mathrm{mM} \mathrm{KCl}, 12 \mathrm{mM} \mathrm{NaHCO}_{3}, 5.5 \mathrm{mM}$ glucose, $\mathrm{pH}$ 6.35), and the platelets were then pelleted again in the presence of $\mathrm{PGE}_{1}$ and apyrase. The platelets were resuspended, and the count was adjusted to $3 \times 10^{8}$ platelets $/ \mathrm{m} l$ with Hepes-Tyrode's buffer $(\mathrm{pH} 7.4)$ containing $0.35 \%$ BSA.

Modulation of platelet cholesterol: Cholesterol depletion was performed by incubation of PRP with $5 \mathrm{mM} \mathrm{M} \beta C D$, which is commonly used to remove cholesterol from cell plasma membranes [36], for $15 \mathrm{~min}$ at $37^{\circ} \mathrm{C}$. In contrast, the cholesterol content in platelets was enriched by incubation of PRP with $0.2 \mathrm{mM}$ cholesterol complexed with $2 \mathrm{mM}$ $\mathrm{M} \beta \mathrm{CD}$, which was prepared as described by Furuchi and Anderson [6]. In the cholesterol/ $\mathrm{M} \beta \mathrm{CD}$ complexs, $\mathrm{M} \beta \mathrm{CD}$ acts as carriers for cholesterol and donate cholesterol to the platelet membrane. For cholesterol repletion, cholesteroldepleted $(\mathrm{M} \beta \mathrm{CD}$-treated) platelets were centrifuged and resuspended in Hepes-Tyrode's buffer ( $\mathrm{pH}$ 6.35) containing $0.5 \mathrm{mM}$ cholesterol (complexed with $\mathrm{M} \beta \mathrm{CD}$ ), and then incubated for $30 \mathrm{~min}$ at $37^{\circ} \mathrm{C}$. These platelets were washed as described above and used for the aggregation experiment and measurement of $\mathrm{Ca}^{2+}$ mobilization.

Measurement of platelet aggregation: Agonist-induced platelet shape change and aggregation was analyzed in the presence of $1 \mathrm{mM} \mathrm{CaCl}_{2}$, unless otherwise indicated, by measuring light transmission through the platelet suspension using a HEMA TRACER (Niko Bioscience Co, Tokyo, Japan) or a CAF-100 (JASCO, Tokyo, Japan) at $37^{\circ} \mathrm{C}$ with constant stirring. For each experiment, the aggregometer was calibrated with Hepes-Tyrode's buffer ( $\mathrm{pH}$ 7.4) for $100 \%$ transmission and with a platelet supension for $0 \%$ transmission. The extent of the aggregation is expressed as a percentage of transmission. Shape change is indicated as a decrease in light transmission through the suspension. It should be noted that the shape change is presented graphically in this paper as a downward deflection in the traces.

Measurement of intracellular $\mathrm{Ca}^{2+}$ mobilization: Platelets in Hepes-Tyrode's buffer ( $\mathrm{pH}$ 6.35) were incubated with $2 \mu \mathrm{M}$ fura-PE3/AM, a fluorescent calcium indicator, for $30 \mathrm{~min}$ at $37^{\circ} \mathrm{C}$. Platelets were then washed and resus- pended as described above. Changes in fluorescence were measured using a CAF-100 (JASCO, Tokyo, Japan) at $37^{\circ} \mathrm{C}$ with constant stirring at excitation wavelengths of 340 and $380 \mathrm{~nm}$ and at an emission wavelength of $500 \mathrm{~nm}$. Fluorescence measurements were converted to $\mathrm{Ca}^{2+}$ concentrations using the equation reported by Grynkiewicz et al. [8] with a $\mathrm{K}_{\mathrm{D}}$ value of $290 \mathrm{nM}$ for fura-PE3 [32]. $\mathrm{F}_{\min }$ and $\mathrm{F}_{\max }$ were determined with each respective platelet preparation.

Protein and cholesterol assay: Protein concentrations were determined using the Bradford method. Platelet cholesterol content was determined with a cholesterol assay kit following the extraction of platelet lipids from the platelet suspension by the method of Folch et al. [5].

Statistical analysis: The results are expressed as the means \pm SEM. The differences between means were evaluated by Student's $t$-test or one-way ANOVA followed by Tukey's test for multiple comparisons, and were considered significant at $P<0.05$.

\section{RESULTS}

Effects of cholesterol depletion on thrombin-induced platelet aggregation: We first examined the effects of membrane cholesterol depletion by treatment of rabbit platelets with $5 \mathrm{mM} \mathrm{M} \beta \mathrm{CD}$, which removed cholesterol from the plasma membranes, on platelet shape change and aggregation induced by thrombin in the presence of $1 \mathrm{mM} \mathrm{CaCl}_{2}$. Stimulation of platelets with thrombin $(0.01-1 \mathrm{unit} / \mathrm{m} l)$ elicited rapid shape change followed by aggregation response in a dose-dependent manner (Fig. 1). The platelet shape change (downward change in trace) induced by thrombin did not differ between $\mathrm{M} \beta \mathrm{CD}$-treated and untreated platelets (Fig. 1A). When platelets were stimulated with the submaximal concentration $(0.1 \mathrm{unit} / \mathrm{m} l)$ of thrombin, $\mathrm{M} \beta \mathrm{CD}$-treated platelets showed a significant decrease in the aggregation response in comparison with the control platelets $(55.7 \pm$ $8.0 \%$ in control and $22.5 \pm 11.1 \%$ in $\mathrm{M} \beta \mathrm{CD}$-treated platelets, $P<0.05, \mathrm{n}=5$ ). However, the extent of platelet aggregation induced by the higher concentration of thrombin (1 unit $/ \mathrm{m} l$ ) was not significantly different between $\mathrm{M} \beta \mathrm{CD}$ treated and control platelets (Fig. 1B).

To confirm that the inhibitory effect of $\mathrm{M} \beta \mathrm{CD}$ on platelet aggregation is due to the depletion of cholesterol in platelets, we examined the effects of cholesterol depletion/repletion on cholesterol content and thrombin-induced aggregation (Fig. 2). In $\mathrm{M} \beta \mathrm{CD}$-treated platelets, the cholesterol content was significantly decreased in comparison with control platelets $(0.47 \pm 0.07 \mathrm{mg}$ in control and $0.20 \pm$ $0.03 \mathrm{mg}$ in $\mathrm{M} \beta \mathrm{CD}$-treated platelets, $P<0.05, \mathrm{n}=5$, in Fig. $2 \mathrm{~A}$ ), in coincidence with the inhibition of aggregation induced by thrombin (Fig. $2 \mathrm{~B}$ ). When $\mathrm{M} \beta \mathrm{CD}$-treated platelets were incubated with cholesterol/M $\beta \mathrm{CD}$ complex to replete platelet cholesterol, the cholesterol content and thrombin-induced aggregation were recovered to control values. These results suggested that the inhibition of platelet aggregation by treatment with $\mathrm{M} \beta \mathrm{CD}$ was due to the depletion of cholesterol in platelets. 
A

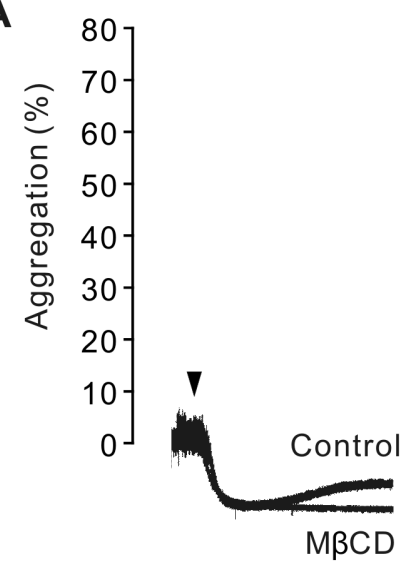
0.01

Thrombin (unit/ml)

B

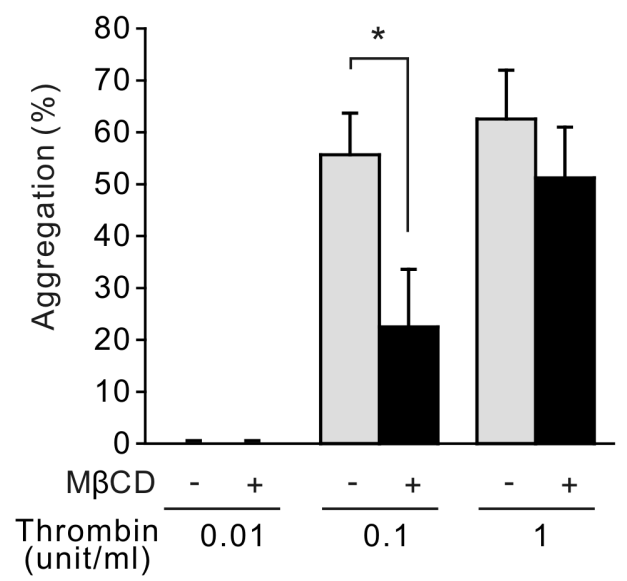

Fig. 1. Effects of cholesterol depletion on thrombin-induced platelet aggregation. Rabbit platelet cholesterol was depleted in response to $\mathrm{M} \beta \mathrm{CD}$ treatment, as described in Materials and Methods. Washed rabbit platelets were stimulated with 0.01-1 unit $/ \mathrm{m} l$ thrombin in the presence of $1 \mathrm{mM} \mathrm{CaCl}_{2}$. A: Representative aggregation curves in control and $\mathrm{M} \beta \mathrm{CD}$-treated platelets. Arrow head indicates the addition of thrombin. B: Bar graph shows the maximal amplitude of aggregation within 10 min after the addition of thrombin.* $P<0.05$ (Student's $t$-test), $\mathrm{n}=5$.

Effects of cholesterol depletion on thrombin-induced $\mathrm{Ca}^{2+}$ mobilization: Since it is widely accepted that elevation of cytosolic free $\mathrm{Ca}^{2+}$ concentrations $\left(\left[\mathrm{Ca}^{2+}\right]_{\mathrm{i}}\right)$ is an essential step in platelet activation induced by most agonists [22], we studied the effects of cholesterol depletion on $\left[\mathrm{Ca}^{2+}\right]_{\mathrm{i}}$ in furaPE3-loaded rabbit platelets. We first observed the effects of cholesterol depletion on thrombin-induced $\mathrm{Ca}^{2+}$ mobilization in the presence of $1 \mathrm{mM} \mathrm{CaCl}_{2}$ (Fig. 3). In control platelets, stimulation with thrombin $(0.01-1 \mathrm{unit} / \mathrm{m} /)$ elic-
A

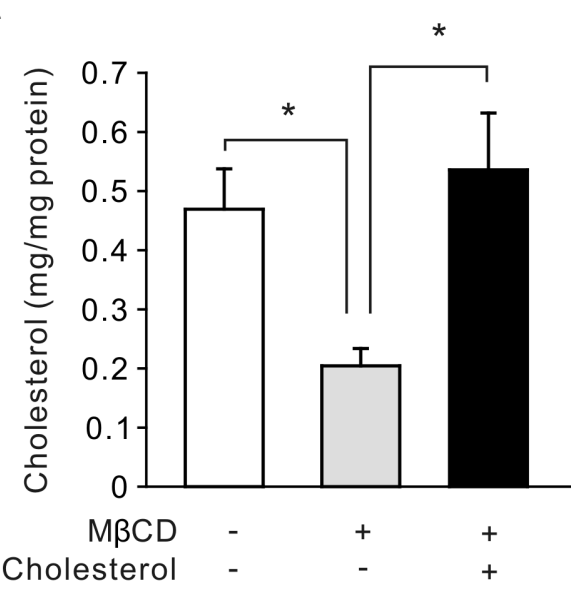

B

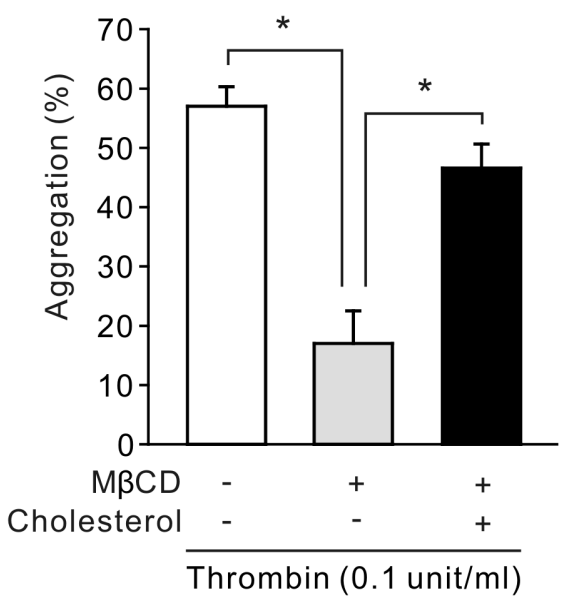

Fig. 2. $\mathrm{M} \beta \mathrm{CD}$ decreases thrombin-induced aggregation in a cholesterol-dependent manner. A: Cholesterol content in control, $\mathrm{M} \beta \mathrm{CD}$-treated, and cholesterol-repleted platelets was assessed as described in Materials and Methods. * $P<0.05$ (Tukey's test), $\mathrm{n}=5$. B: Bar graph shows the maximal amplitude of aggregation within $10 \mathrm{~min}$ after the addition of $0.1 \mathrm{unit} / \mathrm{m} l$ thrombinin in control, $\mathrm{M} \beta \mathrm{CD}$-treated, and cholesterol-repleted platelets in the presence of $1 \mathrm{mM} \mathrm{CaCl}_{2}$. ${ }^{*} P<0.05$ (Tukey's test), $n=5$.

ited an increase in $\left[\mathrm{Ca}^{2+}\right]_{\mathrm{i}}$ in a dose-dependent manner (Fig. 3B). The increase in $\left[\mathrm{Ca}^{2+}\right]_{\mathrm{i}}$ induced by $0.01-1 \mathrm{unit} / \mathrm{m} l$ thrombin was diminished in $\mathrm{M} \beta \mathrm{CD}$-treated platelets in comparison with that in control platelets, although the inhibition was most apparent at lower concentrations of thrombin.

Increases in $\left[\mathrm{Ca}^{2+}\right]_{\mathrm{i}}$ are regulated by the release of $\mathrm{Ca}^{2+}$ from intracellular $\mathrm{Ca}^{2+}$ stores and the influx of $\mathrm{Ca}^{2+}$ across the plasma membrane. In platelets, store-mediated $\mathrm{Ca}^{2+}$ entry, a mechanism regulated by the filling state of the intracellular $\mathrm{Ca}^{2+}$ store, is believed to be an important pathway 
A

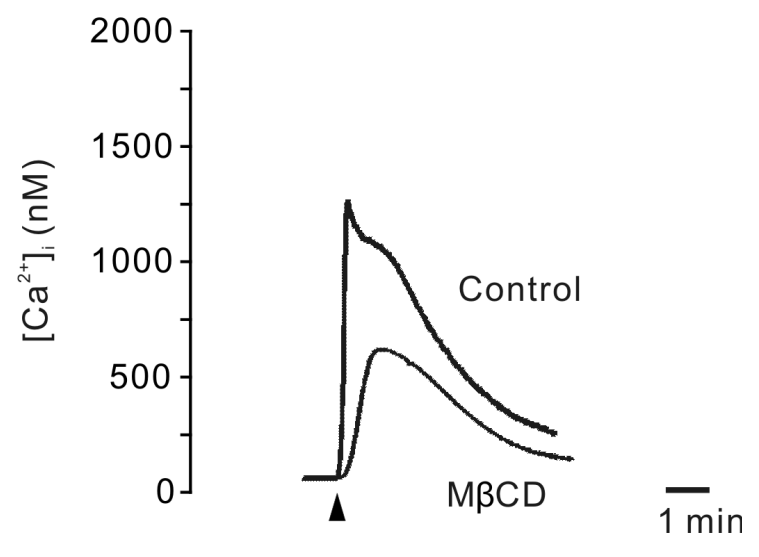

Thrombin 0.1 unit $/ \mathrm{ml}$

B

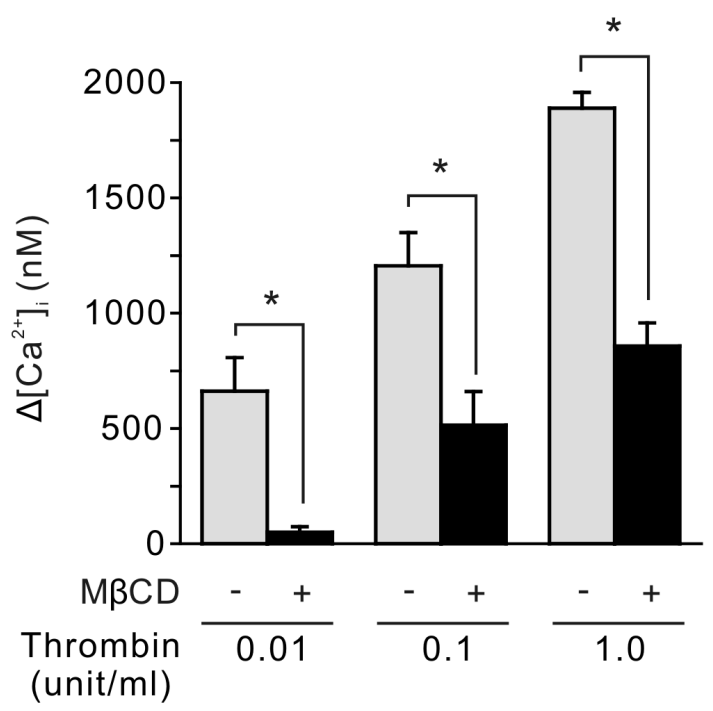

Fig. 3. Effects of cholesterol depletion on thrombin-induced $\mathrm{Ca}^{2+}$ mobilization. Control or $\mathrm{M} \beta \mathrm{CD}$-treated washed rabbit platelets were loaded with Fura-PE3 and stimulated with 0.01$1 \mathrm{unit} / \mathrm{m} l$ thrombin in the presence of $1 \mathrm{mM} \mathrm{CaCl}_{2}$. A: Representative $\mathrm{Ca}^{2+}$ mobilization in control or $\mathrm{M} \beta \mathrm{CD}$-treated platelets induced by $0.1 \mathrm{unit} / \mathrm{m} l$ thrombin. Arrow head indicates the addition of thrombin. B: Bar graph shows maximal amplitude of increase in $\left[\mathrm{Ca}^{2+}\right]_{\mathrm{i}}$ within $5 \mathrm{~min}$ after the addition of thrombin. $* P<0.05$ (Tukey's test), $\mathrm{n}=4$.

for $\mathrm{Ca}^{2+}$ influx [22]. Since cholesterol depletion by treatment with $\mathrm{M} \beta \mathrm{CD}$ inhibited $\mathrm{Ca}^{2+}$ mobilization in rabbit platelets, we examined the effect of the $\mathrm{M} \beta \mathrm{CD}$ treatment on $\mathrm{Ca}^{2+}$ release and $\mathrm{Ca}^{2+}$ influx induced by thrombin (Fig. 4). To observe $\mathrm{Ca}^{2+}$ release from $\mathrm{Ca}^{2+}$ stores, fura-PE3-loaded platelets were stimulated by thrombin for $3 \mathrm{~min}$ in the absence of extracellular $\mathrm{Ca}^{2+}$ (with $1 \mathrm{mM}$ EGTA added), and then $2 \mathrm{mM} \mathrm{CaCl}_{2}$ was added to the extracellular buffer to elicit $\mathrm{Ca}^{2+}$ influx (Fig. 4A). Although cholesterol depletion tends to reduce $\mathrm{Ca}^{2+}$ release from intracellular stores
A

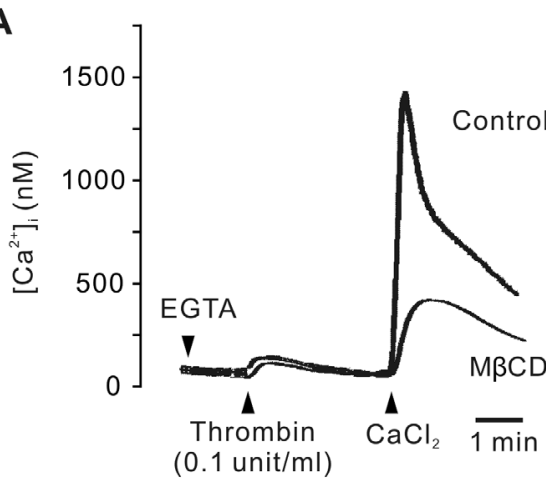

B

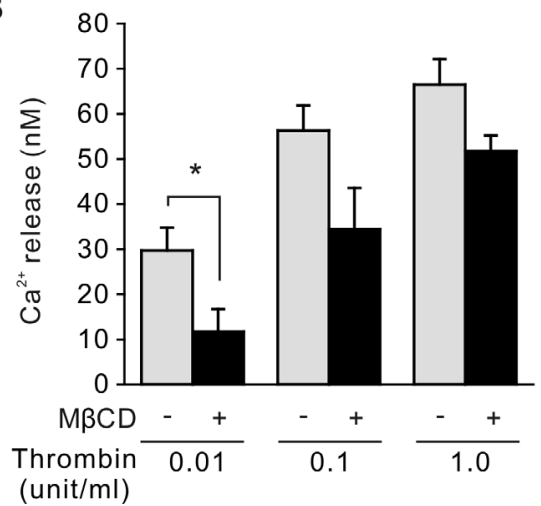

C

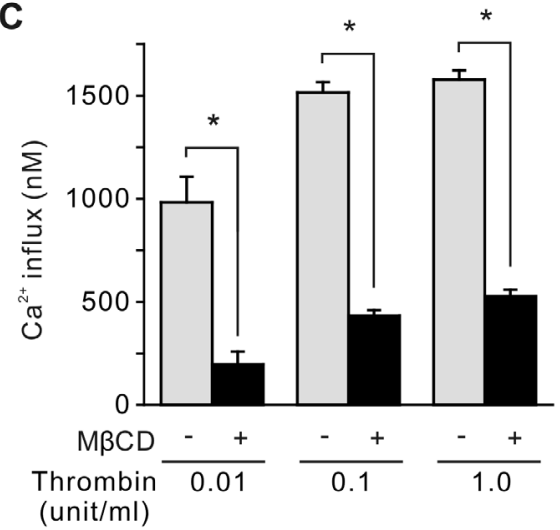

Fig. 4. Cholesterol depletion inhibits $\mathrm{Ca}^{2+}$ influx and $\mathrm{Ca}^{2+}$ release induced by thrombin. A: Representative $\mathrm{Ca}^{2+}$ mobilization in control or $\mathrm{M} \beta \mathrm{CD}$-treated platelets induced by $0.1 \mathrm{unit} /$ $\mathrm{m} l$ thrombin in the absence or presence of extracellular $\mathrm{Ca}^{2+}$. Arrow head indicates the addition of the $1 \mathrm{mM}$ EGTA, 0.1 unit/ $\mathrm{m} l$ thrombin, and $2 \mathrm{mM} \mathrm{CaCl}$. $\mathrm{B}$ : Bar graph shows $\mathrm{Ca}^{2+}$ release from $\mathrm{Ca}^{2+}$ stores induced by thrombin in control or $\mathrm{M} \beta \mathrm{CD}$-treated platelets. $\mathrm{Ca}^{2+}$ release was measured as the maximal amplitude of increase in $\left[\mathrm{Ca}^{2+}\right]_{\mathrm{i}}$ within $3 \mathrm{~min}$ after the addition of $0.1 \mathrm{unit} / \mathrm{m} l$ thrombin in the presence of $1 \mathrm{mM}$ EGTA. * $P<0.05$ (Student's $t$-test), $\mathrm{n}=4$. C: Bar graph shows $\mathrm{Ca}^{2+}$ influx induced by thrombin in control or $\mathrm{M} \beta C D$-treated platelets. $\mathrm{Ca}^{2+}$ influx was measured as the maximal amplitude of increase in $\left[\mathrm{Ca}^{2+}\right]_{\mathrm{i}}$ within $3 \mathrm{~min}$ after the addition of $2 \mathrm{mM}$ $\mathrm{CaCl}_{2}$ in the presence of $1 \mathrm{mM} \mathrm{EGTA}$ and $0.1 \mathrm{unit} / \mathrm{m} l$ thrombin. ${ }^{*} P<0.05$ (Student's $t$-test), $\mathrm{n}=3$. 
A

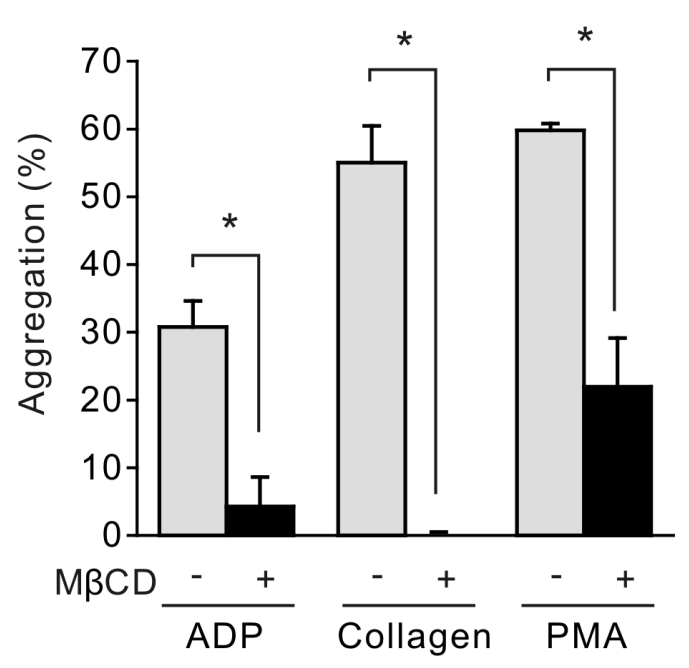

B

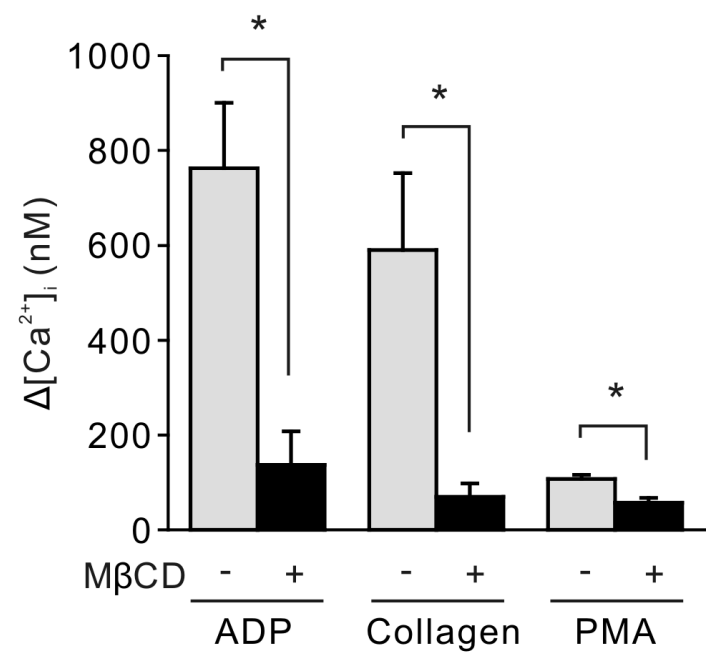

Fig. 5. Effects of cholesterol depletion on platelet activation induced by ADP, collagen, and PMA. A: Maximal amplitude of aggregation within $10 \mathrm{~min}$ after the addition of $30 \mu \mathrm{M}$ ADP or $10 \mu \mathrm{g} / \mathrm{m} l$ collagen, or within $30 \mathrm{~min}$ after the addition of $3 \mu \mathrm{M}$ PMA in control or $\mathrm{M} \beta \mathrm{CD}$-treated platelets in the presence of $1 \mathrm{mM} \mathrm{CaCl}_{2}{ }^{*} P<0.05$ (Student's $t$-test), $\mathrm{n}=3$. B: Maximal amplitude of increase in $\left[\mathrm{Ca}^{2+}\right]_{\mathrm{i}}$ within $10 \mathrm{~min}$ after the addition of $30 \mu \mathrm{M}$ ADP or $10 \mu \mathrm{g} / \mathrm{m} l$ collagen, or within 30 min after the addition of $3 \mu \mathrm{M}$ PMA in control or M $\beta C D-$ treated platelets in the presence of $1 \mathrm{mM} \mathrm{CaCl}_{2}$. $* \mathrm{P}<0.05$ (Student's $t$-test), $\mathrm{n}=3$.

induced by thrombin, significant inhibition of $\mathrm{Ca}^{2+}$ release in comparison with that in control platelets was only observed at $0.01 \mathrm{unit} / \mathrm{m} l$ thrombin $(29.8 \pm 5.0 \mathrm{nM}$ in control and $11.8 \pm 5.0 \mathrm{nM}$ in $\mathrm{M} \beta \mathrm{CD}$-treated platelets, $P<0.05, \mathrm{n}=4$, in Fig. 4B). On the other hand, thrombin-induced $\mathrm{Ca}^{2+}$ influx was significantly inhibited in cholesterol-depleted platelets stimulated with $0.01-1 \mathrm{unit} / \mathrm{m} l$ thrombin (inhibition $(\%) ; 80.7 \pm 4.2,71.4 \pm 0.9$ or $66.5 \pm 2.4$ in $0.01,0.1$ or

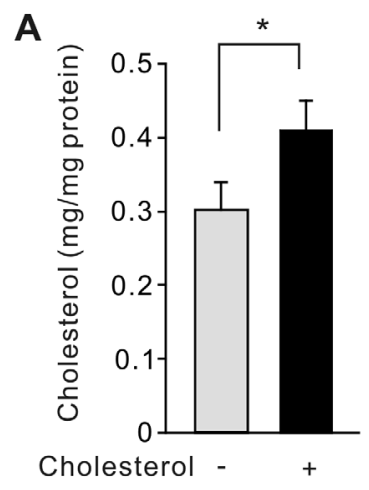

B
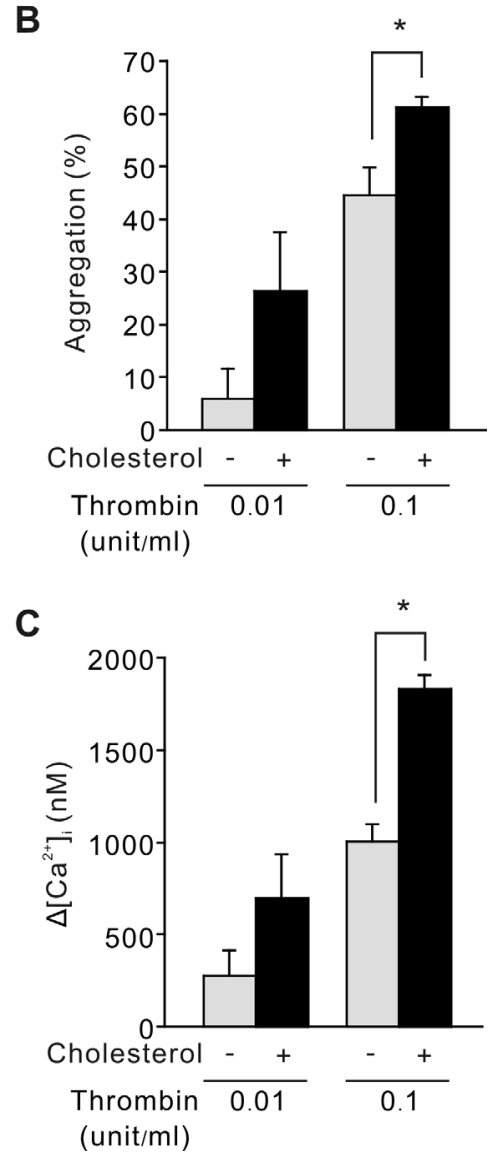

Fig. 6. Cholesterol enrichment augmented platelet activation induced by thrombin. Rabbit platelet cholesterol was enriched by treatment of the cholesterol/M $\beta \mathrm{CD}$ complex, as described in Materials and Methods. A: Cholesterol content in control or cholesterol-enriched platelets. ${ }^{*} P<0.05$ (Student's $t$-test), $\mathrm{n}=5$. B: Maximal amplitude of aggregation within $10 \mathrm{~min}$ after the addition of 0.01 or $0.1 \mathrm{unit} / \mathrm{m} l$ thrombin in control and cholesterol-enriched platelets in the presence of $1 \mathrm{mM} \mathrm{CaCl}_{2}$. * $P<0.05$ (Student's $t$-test), $\mathrm{n}=5$. C: Maximal amplitude of increase in $\left[\mathrm{Ca}^{2+}\right]_{\mathrm{i}}$ within $10 \mathrm{~min}$ after the addition of 0.01 or $0.1 \mathrm{unit} / \mathrm{m} l$ thrombin in control and cholesterol-enriched platelets in the presence of $1 \mathrm{mM} \mathrm{CaCl}_{2}$. ${ }^{*} P<0.05$ (Student's $t$-test), $\mathrm{n}=5$. 
$1 \mathrm{unit} / \mathrm{m} l$ thrombin, $\mathrm{n}=3$, in Fig. $4 \mathrm{C}$ ). These results suggest that thrombin-induced $\mathrm{Ca}^{2+}$ influx is more sensitive to cholesterol depletion than $\mathrm{Ca}^{2+}$ release from intracellular stores.

Effects of cholesterol depletion on platelet activation induced by ADP, collagen, and PMA: We examined the effects of cholesterol depletion on platelet aggregation and $\mathrm{Ca}^{2+}$ mobilization induced by other physiological agonists, ADP, and collagen (Fig. 5). ADP (1-30 $\mu \mathrm{M})$ or collagen $(0.3-10 \mu \mathrm{g} / \mathrm{m} l)$ induced aggregation and $\mathrm{Ca}^{2+}$ mobilization in rabbit platelets in a dose-dependent manner (data not shown). The platelet aggregation induced by $30 \mu \mathrm{M}$ ADP or $10 \mu \mathrm{g} / \mathrm{m} l$ collagen was significantly diminished by cholesterol depletion (ADP; $30.8 \pm 3.8 \%$ in control and $4.3 \pm 4.3 \%$ in $\mathrm{M} \beta \mathrm{CD}$-treated platelets, collagen; $55.0 \pm 5.4 \%$ in control and $0 \pm 0 \%$ in $\mathrm{M} \beta \mathrm{CD}$-treated platelets, $P<0.05, \mathrm{n}=3$, in Fig. $5 \mathrm{~A})$. In agreement with the results for platelet aggregation, we observed that $\mathrm{Ca}^{2+}$ mobilization induced by ADP or collagen was inhibited in cholesterol-depleted platelets (Fig. 5B). We also examined the effects of cholesterol depletion on platelet activation induced by PMA, a potent protein kinase $\mathrm{C}$ (PKC) activator [16]. Although treatment of control platelets with $3 \mu \mathrm{M}$ PMA evoked platelet aggregation, PMA caused only a small increase in $\left[\mathrm{Ca}^{2+}\right]_{\mathrm{i}}$ in comparison with that induced by thrombin, ADP, or collagen. In cholesterol-depleted platelets, PMA-induced aggregation was significantly inhibited compared with that in control platelets $(59.8 \pm 1.0 \%$ in control and $21.9 \pm 7.2 \%$ in $\mathrm{M} \beta \mathrm{CD}$-treated platelets, $P<0.05$, n=3, in Fig. 5A).

Effects of cholesterol enrichment on platelet activation: Finally, we examined the effects of cholesterol enrichment in platelets on aggregation and $\mathrm{Ca}^{2+}$ mobilization induced by thrombin (Fig. 6). Incubation of PRP with cholesterol/ $\mathrm{M} \beta \mathrm{CD}$ complexes significantly increased the cholesterol content in rabbit platelets in comparison with that in untreated platelets $(0.30 \pm 0.03 \mathrm{mg}$ in control and $0.41 \pm$ $0.04 \mathrm{mg}$ in $\mathrm{M} \beta \mathrm{CD}$-treated platelets, $P<0.05, \mathrm{n}=5$, in Fig. $6 \mathrm{~A})$. Platelet aggregation and $\mathrm{Ca}^{2+}$ mobilization induced by $0.1 \mathrm{unit} / \mathrm{m} l$ thrombin were significantly augmented in the cholesterol-enriched platelets (aggregation; $44.6 \pm 5.8 \%$ in control and $61.2 \pm 2.2 \%$ in cholesterol-enriched platelets, $\mathrm{Ca}^{2+}$ mobilization; $944.5 \pm 97.4 \mathrm{nM}$ in control and $1799.0 \pm$ $88.1 \mathrm{nM}$ in cholesterol-enriched platelets, $P<0.05, \mathrm{n}=5$, in Fig. 6B and 6C). A similar tendency was also observed at $0.01 \mathrm{unit} / \mathrm{m} l$ thrombin. These results suggest that the cholesterol content in platelets is a critical determinant in the regulation of platelet activation.

\section{DISCUSSION}

In the present study, we investigated the effects of in vitro modulation of cholesterol content in rabbit platelets on the platelet aggregation and $\mathrm{Ca}^{2+}$ mobilization induced by platelet-aggregating agonists. We showed that platelet aggregation induced by physiological agonists such as thrombin, ADP, and collagen is inhibited by the treatment of platelets with $\mathrm{M} \beta \mathrm{CD}$, which is commonly used to remove cholesterol from cell plasma membranes [36]. The inhibition of platelet aggregation by treatment with $\mathrm{M} \beta \mathrm{CD}$ is due to the decrease in the platelet cholesterol content, since the inhibition of thrombin-induced aggregation is significantly restored by cholesterol repletion by incubation of the $\mathrm{M} \beta \mathrm{CD}$-treated platelets with cholesterol/M $\beta \mathrm{CD}$ complexes. Furthermore, platelet shape change induced by thrombin or platelet aggregation induced by higher concentrations of thrombin (1 unit/ $\mathrm{m} l$ ) was not affected by treatment of $\mathrm{M} \beta \mathrm{CD}$, suggesting that the inhibitory effect of $\mathrm{M} \beta \mathrm{CD}$ is not due to the nonspecific toxic effect on platelets.

Consistent with a report regarding human platelets [6], higher concentrations of thrombin $(1 \mathrm{unit} / \mathrm{m} l)$ elicited platelet aggregation to a similar extent in both cholesteroldepleted and control platelets. This phenomenon might reflect the distinct sensitivity of thrombin receptors to cholesterol depletion, as in the case of ADP-induced human platelet activation, in which $\mathrm{ADP}$ receptor $\mathrm{P} 2 \mathrm{X}_{1}$ and $\mathrm{P} 2 \mathrm{Y}_{12}$, but not $\mathrm{P}_{2} \mathrm{Y}_{1}$, exhibit dependencies on platelet cholesterol $[20,31]$. In human platelets, thrombin activates a family of protease-activated receptors (PARs) such as PAR-1, -3, and -4 , in which PAR-1 or PAR-4 mediates platelet responses at low or high concentrations of thrombin, respectively [10]. In rabbit platelets, expression of PAR-3 had been reported [11]. However, the thrombin-induced signaling pathways that promote rabbit platelet aggregation are essentially unknown. The expression pattern of the PARs and cholesterol dependencies of PARs must be elucidated in rabbit platelets.

A pivotal role of intracellular $\mathrm{Ca}^{2+}$ mobilization in ADP secretion, $\mathrm{TXA}_{2}$ generation, and platelet aggregation has been reported [22, 24, 27]. In agreement with the results of our aggregation experiment and previous reports regarding human platelets $[6,7,30]$, cholesterol depletion reduced the increases in $\left[\mathrm{Ca}^{2+}\right]_{\mathrm{i}}$ induced by thrombin, ADP, and collagen, thus indicating the important role of cholesterol in $\mathrm{Ca}^{2+}$ mobilization mechanisms. Unlike the aggregation experiments, treatment of $\mathrm{M} \beta \mathrm{CD}$ significantly suppressed $\mathrm{Ca}^{2+}$ mobilization induced by high concentration of thrombin $(1 \mathrm{unit} / \mathrm{m} l)$. It is likely that increase in $\left[\mathrm{Ca}^{2+}\right]_{\mathrm{i}}$ induced by $1 \mathrm{unit} / \mathrm{m} l$ thrombin in the presence of $\mathrm{M} \beta \mathrm{CD}$ is still high enough to elicit nearly full aggregation response. Increases in $\left[\mathrm{Ca}^{2+}\right]_{\mathrm{i}}$ in platelets are regulated by two pathways: the release of $\mathrm{Ca}^{2+}$ from intracellular $\mathrm{Ca}^{2+}$ stores and the influx of $\mathrm{Ca}^{2+}$ across the plasma membrane. We showed that $\mathrm{Ca}^{2+}$ influx elicited by the addition of $\mathrm{CaCl}_{2}$ to extracellular medium after thrombin stimulation is inhibited in cholesterol-depleted platelets in comparison with control platelets at all the concentrations tested (0.01-1 unit $/ \mathrm{m} l$ thrombin). A similar suppression of the $\mathrm{Ca}^{2+}$ influx pathway by cholesterol depletion has also been reported in human platelets [2]. In contrast, significant inhibition of $\mathrm{Ca}^{2+}$ release from $\mathrm{Ca}^{2+}$ stores in cholesterol-depleted platelets was only observed when the platelets were stimulated with a low concentration of thrombin $(0.01 \mathrm{unit} / \mathrm{m} l)$. These results suggest that thrombin-induced $\mathrm{Ca}^{2+}$ influx is more sensitive to cholesterol depletion by $\mathrm{M} \beta \mathrm{CD}$-treatment than thrombin-induced $\mathrm{Ca}^{2+}$ release from intracellular stores. It has been suggested 
that the coupling of type II 1,4,5-triphosphate receptor $\left(\mathrm{IP}_{3} \mathrm{RII}\right)$ to transient receptor potential canonical 1 (TRPC1), which is involved in store-mediated $\mathrm{Ca}^{2+}$ entry in human platelets, is reduced following cholesterol depletion [2]. This inhibition of coupling between $\mathrm{IP}_{3} \mathrm{RII}$ and hTRPC1 might be involved in the inhibition of $\mathrm{Ca}^{2+}$ influx by cholesterol depletion in rabbit platelets.

To analyze the involvement of platelet cholesterol in the $\mathrm{Ca}^{2+}$-independent platelet activation pathway, we also examined the effects of $\mathrm{M} \beta \mathrm{CD}$ treatment on platelet activation induced by PMA, which directly activates intracellular $\mathrm{PKC}$ and results in ADP secretion and platelet aggregation without apparent $\mathrm{Ca}^{2+}$ mobilization [4, 19]. It seems that PMA-induced aggregation is largely independent of $\mathrm{Ca}^{2+}$ mobilization, since although $3 \mu \mathrm{M}$ PMA elicited platelet aggregation to a similar extent as that induced by $0.1 \mathrm{unit} / \mathrm{m} l$ thrombin or $10 \mu \mathrm{g} / \mathrm{m} l$ collagen, PMA caused only a small increase in $\left[\mathrm{Ca}^{2+}\right]_{\mathrm{i}}$. We have shown that PMA-induced aggregation is partially inhibited by cholesterol depletion in rabbit platelets. It is likely that the $\mathrm{Ca}^{2+}$-independent pathways downstream from PKC are inhibited by cholesterol depletion in platelets, in addition to the inhibition of $\mathrm{Ca}^{2+}$ mobilization. In contrast, in human platelets, PMA-induced aggregation and ADP secretion are not sensitive to treatment with $\mathrm{M} \beta \mathrm{CD}$ [4]. The species difference in platelet activation mechanisms such as the involvement of ADP/ $\mathrm{TXA}_{2}$ and the role of PKC in platelet activation must be elucidated in future studies.

Finally, we demonstrated that the platelet aggregation and $\mathrm{Ca}^{2+}$ mobilization induced by $0.1 \mathrm{unit} / \mathrm{ml}$ thrombin are significantly augmented in cholesterol-enriched rabbit platelets, in which cholesterol content was increased to $136 \%$ of control platelets. This result is in accordance with the observation that platelets from hypercholesterolemic humans and rabbits have increased cholesterol content (approximately $150 \%$ of control platelets) and hypersensitivity to platelet-aggregating agonists $[3,21,25,28]$. Together, our results suggested that the cholesterol content in platelets is critical to the regulation of platelet activation both in vitro and in vivo.

It has been reported that endogenous agonists, $\mathrm{TXA}_{2}$ and ADP, plays a crucial role in collagen-induced activation in human platelets, and in vitro cholesterol depletion reduces release of the endogenous agonists $[4,7,27,30]$. We showed that, in rabbit platelets, cholesterol depletion not only decreased collagen-induced activation, but also suppressed platelet activation induced by ADP. It is likely that modulation of cholesterol content in rabbit platelets affect the release of endogenous agonists and/or activation mechanisms elicited by the released endogenous agonists. Further research focused on the roles of endogenous agonists is needed to clarify the precise mechanisms controlling the rabbit platelet activation by modulation of cholesterol contents.

In summary, we have shown that (1) cholesterol depletion in rabbit platelets by the treatment with $\mathrm{M} \beta \mathrm{CD}$ decreases the platelet aggregation and $\mathrm{Ca}^{2+}$ mobilization induced by thrombin, (2) the $\mathrm{Ca}^{2+}$ influx pathway is more sensitive to cholesterol depletion than $\mathrm{Ca}^{2+}$ release from $\mathrm{Ca}^{2+}$ stores, (3) cholesterol depletion also inhibits ADP- or collageninduced platelet activation, and (4) cholesterol enrichment augments thrombin-induced platelet activation. These findings suggest that cholesterol plays a critical role in regulating rabbit platelet activation, and provides fundamental information regarding hypercholesterolemia-mediated effects on cells in the rabbit model.

\section{REFERENCES}

1. Bodin, S., Giuriato, S., Ragab, J., Humbel, B.M., Viala, C., Vieu, C., Chap, H. and Payrastre, B. 2001. Production of phosphatidylinositol 3,4,5-trisphosphate and phosphatidic acid in platelet rafts: evidence for a critical role of cholesterolenriched domains in human platelet activation. Biochemistry 40: $15290-15299$.

2. Brownlow, S.L., Harper, A.G., Harper, M.T. and Sage, S.O. 2004. A role for hTRPC1 and lipid raft domains in store-mediated calcium entry in human platelets. Cell Calcium 35: 107113.

3. Dalal, K.B., Ebbe, S., Mazoyer, E., Carpenter, D. and Yee, T. 1990. Biochemical and functional abnormalities in hypercholesterolemic rabbit platelets. Lipids 25: 86-92.

4. Ezumi, Y., Kodama, K., Uchiyama, T. and Takayama, H. 2002. Constitutive and functional association of the platelet collagen receptor glycoprotein VI-Fc receptor $\gamma$-chain complex with membrane rafts. Blood 99: 3250-3255.

5. Folch, J., Lees, M. and Sloane Stanley, G.H. 1957. A simple method for the isolation and purification of total lipides from animal tissues. J. Biol. Chem. 226: 497-509.

6. Furuchi, T. and Anderson, R.G. 1998. Cholesterol depletion of caveolae causes hyperactivation of extracellular signal-related kinase (ERK). J. Biol. Chem. 273: 21099-21104.

7. Grgurevich, S., Krishnan, R., White, M.M. and Jennings, L.K. 2003. Role of in vitro cholesterol depletion in mediating human platelet aggregation. J. Thromb. Haemost. 1: 576-586.

8. Grynkiewicz, G., Poenie, M. and Tsien, R.Y. 1985. A new generation of $\mathrm{Ca}^{2+}$ indicators with greatly improved fluorescence properties. J. Biol. Chem. 260: 3440-3450.

9. Hochgraf, E., Levy, Y., Aviram, M., Brook, J.G. and Cogan, U. 1994. Lovastatin decreases plasma and platelet cholesterol levels and normalizes elevated platelet fluidity and aggregation in hypercholesterolemic patients. Metabolism 43: 11-17.

10. Kahn, M.L., Nakanishi-Matsui, M., Shapiro, M.J., Ishihara, H. and Coughlin, S.R. 1999. Protease-activated receptors 1 and 4 mediate activation of human platelets by thrombin. J. Clin. Invest. 103: 879-887.

11. Khan, T.A., Bianchi, C., Voisine, P., Sandmeyer, J., Feng, J. and Sellke, F.W. 2005. Aprotinin inhibits protease-dependent platelet aggregation and thrombosis. Ann. Thorac. Surg. 79: $1545-1550$.

12. Kolodgie, F.D., Katocs, A.S. Jr, Largis, E.E., Wrenn, S.M., Cornhill, J.F., Herderick, E.E., Lee, S.J. and Virmani, R. 1996. Hypercholesterolemia in the rabbit induced by feeding graded amounts of low-level cholesterol. Methodological considerations regarding individual variability in response to dietary cholesterol and development of lesion type. Arterioscler. Thromb. Vasc. Biol. 16: 1454-1464.

13. Kramer, R.M., Jakubowski, J.A., Vaillancourt, R. and Deykin, D. 1982. Effect of membrane cholesterol on phospholipid 
metabolism in thrombin-stimulated platelets. Enhanced activation of platelet phospholipase(s) for liberation of arachidonic acid. J. Biol. Chem. 257: 6844-6849.

14. Lacoste, L., Lam, J.Y., Hung, J., Letchacovski, G., Solymoss, C.B. and Waters, D. 1995. Hyperlipidemia and coronary disease. Correction of the increased thrombogenic potential with cholesterol reduction. Circulation 92: 3172-3177.

15. Lindemann, S., Kramer, B., Seizer, P. and Gawaz, M. 2007. Platelets, inflammation and atherosclerosis. J. Thromb. Haemost. 5: 203-211.

16. Nishizuka, Y. 1988. The molecular heterogeneity of protein kinase $\mathrm{C}$ and its implications for cellular regulation. Nature 334: 661-665.

17. Notarbartolo, A., Daví, G., Averna, M., Barbagallo, C.M., Ganci, A., Giammarresi, C., La Placa, F.P. and Patrono, C. 1995. Inhibition of thromboxane biosynthesis and platelet function by simvastatin in type IIa hypercholesterolemia. Arterioscler. Thromb. Vasc. Biol. 15: 247-251.

18. Osamah, H., Mira, R., Sorina, S., Shlomo, K. and Michael, A. 1997. Reduced platelet aggregation after fluvastatin therapy is associated with altered platelet lipid composition and drug binding to the platelets. Br. J. Clin. Pharmacol. 44: 77-83.

19. Pollock, W.K., Rink, T.J. and Irvine, R.F. 1986. Liberation of $\left[{ }^{3} \mathrm{H}\right]$ arachidonic acid and changes in cytosolic free calcium in fura-2-loaded human platelets stimulated by ionomycin and collagen. Biochem. J. 235: 869-877.

20. Quinton, T.M., Kim, S., Jin, J. and Kunapuli, S.P. 2005. Lipid rafts are required in $\mathrm{G} \alpha_{i}$ signaling downstream of the P2Y12 receptor during ADP-mediated platelet activation. J. Thromb. Haemost. 3: 1036-1041.

21. Ravindran, R. and Krishnan, L.K. 2007. Increased platelet cholesterol and decreased percentage volume of platelets as a secondary risk factor for coronary artery disease. Pathophysiol. Haemost. Thromb. 36: 45-51.

22. Rink, T.J. and Sage, S.O. 1990. Calcium signaling in human platelets. Annu. Rev. Physiol. 52: 431-449.

23. Russell, J.C. and Proctor, S.D. 2006. Small animal models of cardiovascular disease: tools for the study of the roles of metabolic syndrome, dyslipidemia, and atherosclerosis. Cardiovasc. Pathol. 15: 318-330.

24. Sargeant, P. and Sage, S.O. 1994. Calcium signalling in platelets and other nonexcitable cells. Pharmacol. Ther. 64: 395443.
25. Sener, A., Ozsavci, D., Oba, R., Demirel, G.Y., Uras, F. and Yardimci, K.T. 2005. Do platelet apoptosis, activation, aggregation, lipid peroxidation and platelet-leukocyte aggregate formation occur simultaneously in hyperlipidemia? Clin. Biochem. 38: 1081-1087.

26. Shattil, S.J., Anaya-Galindo, R., Bennett, J., Colman, R.W. and Cooper, R.A. 1975. Platelet hypersensitivity induced by cholesterol incorporation. J. Clin. Invest. 55: 636-643.

27. Smith, J.B., Selak, M.A., Dangelmaier, C. and Daniel, J.L. 1992. Cytosolic calcium as a second messenger for collageninduced platelet responses. Biochem. J. 288: 925-929.

28. Son, D.J., Lee, H.W., Shin, H.W., Lee, J.J., Yoo, H.S., Kim, T.J., Yun, Y.P. and Hong, J.T. 2008. Enhanced release of sphingosine-1-phosphate from hypercholesterolemic platelets: role in development of hypercholesterolemic atherosclerosis. Prostaglandins Leukot. Essent. Fatty Acids 78: 383-390.

29. Steinberg, D. 2002. Atherogenesis in perspective: hypercholesterolemia and inflammation as partners in crime. Nat. Med. 8: 1211-1217.

30. van Lier, M., Verhoef, S., Cauwenberghs, S., Heemskerk, J.W., Akkerman, J.W. and Heijnen, H.F. 2008. Role of membrane cholesterol in platelet calcium signalling in response to VWF and collagen under stasis and flow. Thromb. Haemost. 99: $1068-1078$.

31 Vial, C., Fung, C.Y., Goodall, A.H., Mahaut-Smith, M.P. and Evans, R.J. 2006. Differential sensitivity of human platelet $\mathrm{P}_{2} \mathrm{X}_{1}$ and $\mathrm{P}_{2} \mathrm{Y}_{1}$ receptors to disruption of lipid rafts. Biochem. Biophys. Res. Commun. 343: 415-419.

32. Vorndran, C., Minta, A. and Poenie, M. 1995. New fluorescent calcium indicators designed for cytosolic retention or measuring calcium near membranes. Biophys. J. 69: 2112-2124.

33. Wagner, D.D. and Burger, P.C. 2003. Platelets in inflammation and thrombosis. Arterioscler. Thromb. Vasc. Biol. 23: 21312137.

34. Watanabe, Y. 1980. Serial inbreeding of rabbits with hereditary hyperlipidemia (WHHL-rabbit). Atherosclerosis 36: 261268.

35. Yanni, A.E. 2004. The laboratory rabbit: an animal model of atherosclerosis research. Lab. Anim. 38: 246-256.

36. Zidovetzki, R. and Levitan, I. 2007. Use of cyclodextrins to manipulate plasma membrane cholesterol content: evidence, misconceptions and control strategies. Biochim. Biophys. Acta 1768: $1311-1324$. 